\title{
Association Between Ethnicity and Dietary Behaviors in Chinese Han and Hui Nationalities
}

\begin{abstract}
Abdelhadi Halawa ${ }^{12}$, Shao-wei $\mathrm{Ai}^{34}, \&$ Zheng $\mathrm{Ma}^{56}$
This cross-sectional study examined the association between sociodemographic characteristics, ethnocultural identity, dietary habits, and related health behaviors of the Chinese Han and Hui populations. Participants in the study were 662 male and female adults randomly selected from four Chinese cities in three provinces. A modified 23question self-administered questionnaire was employed to collect data from the respondents pertaining to their ethnic group affirmation, demographic characteristics, dietary habits, food consumption patterns, and related health practices. A $t$-test was conducted to determine interactions between the parametric variables and Chi-square analyses were conducted to determine interactions between the nonparametric variables. Hans were more likely to have a higher level of education than Huis, Hans were more likely to drink water frequently vs. all other non-water drinks than Huis, both Hans and Huis were more likely to consume their last meal of the day between 17:00-24:00 PM, Huis were more likely to shop for fruits frequently, eat breakfast regularly, cook healthy food (all the time), and bake food more and fry it less frequently, married couples were more likely consume breakfast and shop for fruits more regularly than their single counterparts who were more likely to be have a higher level of education. The level of education does not appear to translate into adopting a healthier dietary behavior such consuming daily breakfast on a regular basis by the more educated single respondents. There is a need to develop both short-term and long-term strategies aimed at further improving the dietary habits and related health practices of both ethnic groups. However, there is a need for the Hans to focus more on improving 6 out of the 8 dietary factors such as consuming regular daily breakfast, and for the Huis to focus more on improving the level of education and 3 out of the 8 dietary factors. Keywords: Chinese food culture, Demographic characteristics, Dietary behaviors, dietary-related health practices, Food consumption patterns, Han and Hui Ethnic Nationalities.
\end{abstract}

\section{Introduction}

Among the 56 officially recognized ethnic groups in China, the Han and Hui Nationalities are two of the most significant ethnic groups. They have distinct cultural identities, distinguishing dietary habits and lifestyle. Dietary habits are strongly influenced by geography, cultures, socioeconomic status, and traditional eating behaviors. For various reasons, even within one country or culture, people usually prepare and consume a variety of food ingredients for different economic reasons, religious beliefs, energy needs, and utilize food as a prophylactic against diseases. Moreover, people living in different cultures obtain and consume their food from different sources to survive and maintain good health. Therefore, dietary habits and food consumption patterns are both cultural traditions and biological process rather than merely physiological and biochemical processes (Damgaci, 2016; Ma, 2015). Research by Kittler, Sucher, and Nelms (2012)

${ }^{1}$ Corresponding Author, National High-End Foreign Expert and Lead Researcher

The Key Research Institute of Yellow River Civilization and Sustainable Development

Henan University, Kaifeng, Henan Province, China

${ }^{2}$ Associate Professor, Millersville University, Millersville, PA, USA

E-mail address: ahalawa@ millersville.edu

Phone: (717) 871-4291

${ }^{3}$ The Key Research Institute of Yellow River Civilization and Sustainable Development

${ }^{4}$ Associate Professor, Henan University, Kaifeng, Henan Province, China

E-Mail Address: aishaowei@163.com

${ }^{5}$ The Key Research Institute of Yellow River Civilization and Sustainable

${ }^{6}$ Associate Professor, Henan University, Kaifeng, Henan Province, China

E-Mail Address: mazhengyx@163.com 
described food habits or food culture as the traditional ways by which people consume food, including how their food is cultivated; purchased; warehoused, prepared; and the way food is served and to whom is presented. Kittler et al. (2012) further suggested that the significance of this process is unique to humankind, as food functions differ according to each culture in classifying food to reflect its priorities and identity. Food habits may also be employed as an important, or even a determining cultural criterion in a group of people who share the same food denoting their eating patterns, cooking style, and behaviors toward food. However, even within the same culture, dietary behaviors do not necessarily have to be homogenous. As a rule, they are not the same due to sociocultural phenomena such as diversity in food sources, demographics, religious beliefs, and socioeconomic strata (Chang, 1977). It is accepted a common social norm that people with different cultural or socioeconomic backgrounds would usually have different diets and broadly consume food differently with a variety of choices. Likewise, people with different religious beliefs have different dietary rules that regulate what is permissible to eat and what is not (Valera, 2008).

Comparable to other ancient civilizations such as ancient Egypt, Chinese food consumption is shaped by certain ancient health beliefs and homeopathic restorative benefits that are associated with each kind of food consumed. In the Chinese culture, this food health and medicinal beliefs have significant effects on how the food is purchased, prepared, and consumed. The prevailing concept about food consumption in China is that food preparation, as well as the kind and amount of food consumed by an individual, is intimately relevant to that individual's overall health and longevity. In general terms, food does not only affect one's physical and emotional well-being, but also the selection of the appropriate food at a particular time must also be dependent upon one's overall health condition at a particular stage of life such as being elderly. Food, therefore, is also considered as part of the traditional Chinese medicine utilized to achieve health maintenance, as well as healing of a myriad of maladies. Consequently, the regulation of one's diet is a constant exercise in disease prevention and treatment that has been practiced for centuries. Chang (1977) argued that Chinese people are especially preoccupied with food. Chang (1977) further explained that food is placed at the center of, or at least, amalgamates with or symbolizes many social interactions and health behaviors of the Chinese people. For thousands of years, in the Chinese culture, there is a deep-rooted belief that an illness is thought to be related to a bodily imbalance or deficiency between the negative energy named "yin" and positive energy named "yang" that can be remedied by a prescribed dietary intake such as herbs and special ingredient soups and multi-grain porridge. Furthermore, the Chinese people believe that it is important to maintain one's good healthy condition through sustaining a good balance between the five elements of well-being through "wuxing", a Chinese term for "five processes". "Wuxing" refers to a quintuple theoretical model of thinking. They include Metal (Jin), Wood (Mu), Water (Shui), Fire (Huo), and Earth (Tu). (Henderson, 2002; Lin, 2000; Roberts, 1999).

Dietary habits in China is influenced by its vast geographical diversity and a widely manifold agricultural landscape, as well as its considerable mélange of diverse ethnic groups (Wang, 2005). Among these major ethnic groups is the Hui Nationality (Chinese: 回族國). The Hui Nationality is one of the largest ethnic minority groups in China. The name "Hui" is derived from the Mandarin Chinese referring to "foreigners" or "outlanders". This name was used for the first time during the Yuan Dynasty (1276-1368 AD) to designate the Hui people as foreigners who settled in China migrating from Central Asia, Persia, Turkey, and the Arab Muslim Middle Eastern Region (Gladney, 1998; Lipman, 1998). Currently, a significant population of the Hui communities resides in the Ningxia Hui Autonomous Region (NHAR) located in the northwest of China. Nearly $20 \%$ of the entire Hui population in China inhabits Ningxia (Parker, 2016). Other Hui communities are similarly concentrated in Xinjiang, Gansu, Qinghai, Hebei, Shandong, Henan, and Yunnan Provinces (Figure 1). 


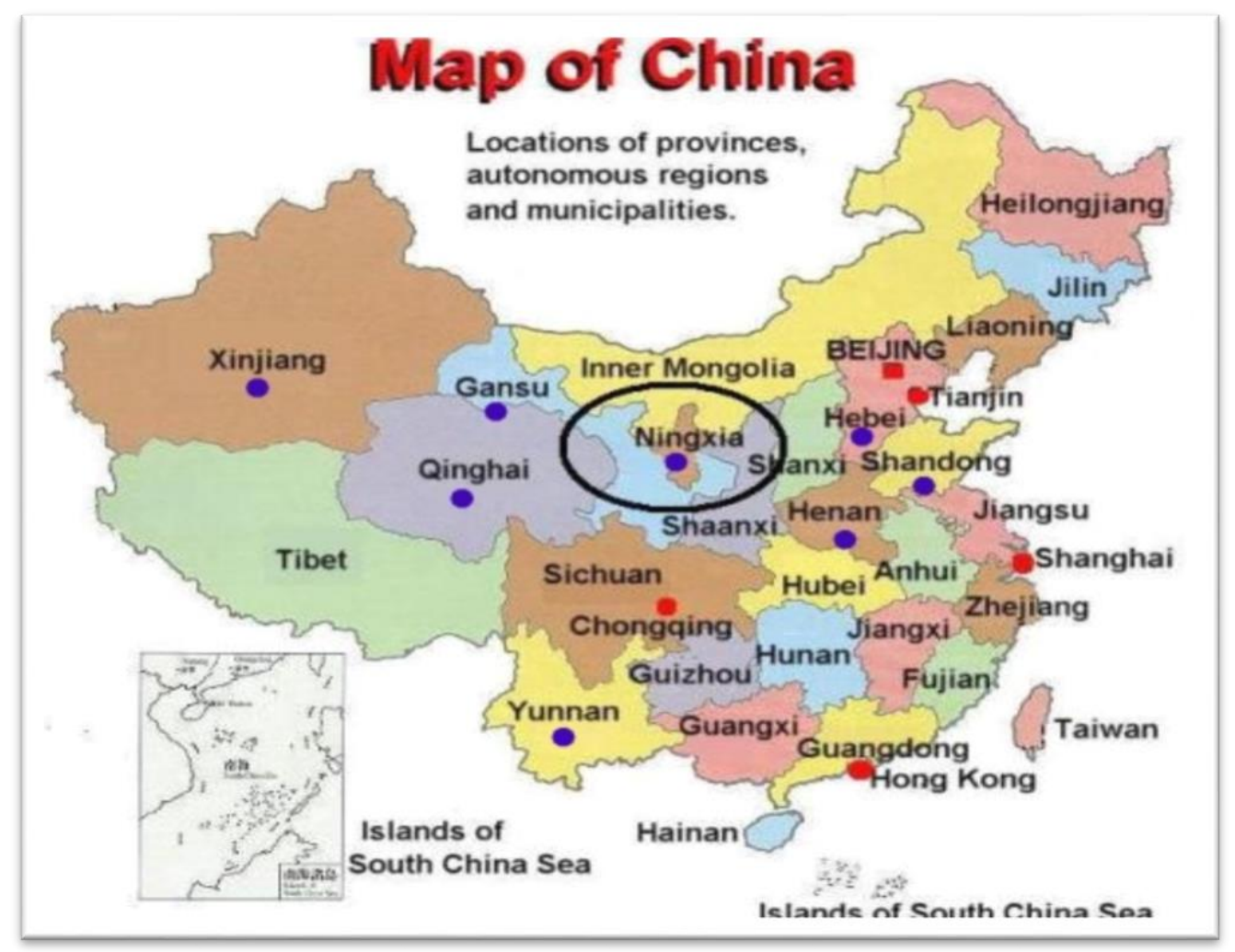

Figure 1. Map of the Hui concentrated areas in China marked by blue dots, and the locations of the three Provinces where the data were collected. Adapted from Word Press (2009).

The progenitors of the Chinese Hui were Muslim merchants and sailors, as well as Persian traders and Central Asian herdsmen migrated to China during the Tang Dynasty between the period of 618 and 905 AD. Migrant Huis settled down in the southwestern, outlying northwestern, and the Yellow River Valley Plains. Eventually, they married Han women and had posterities who became the present-day Hui (Olson, 1998). The Hui people maintain a Sino-Muslim identity; however, they also have their own distinctive dietary practices and a special style of cooking known as "halal food" or "lawful food" (Chinese: 清真食品, Qing Zhen) that has been observed by Muslims for nearly 1400 years as a dietary requirement of all adherents of Sunni Islam. The Muslim ancestors of the Chinese Hui who journeyed to China through the maritime and overland Silk Road routes did not only transport their Islamic religion into China, but also their traditional "halal food" butchering of animals for consumption, their unique cooking styles, and other Islamic dietary practices (Newman, 2004).

According to Feagin and Feagin (2007), an ethnic minority group is a group of people who are distinguished from another dominant group of people who constitute the preponderance of the total population. The main differences that set them apart, including their physical traits, distinct cultural characteristics such as language; religious beliefs; food choices; dress costumes; and social traditions such as marriage and burial ceremonies (Faltis, 2014; Tarman \& Gürel, 2017). Together with the Han majority Nationality, there are 56 officially recognized ethnic groups in China. Each group has its own unique food ingredients, cooking styles, dietary habits, and food health restorative beliefs that were developed throughout the centuries. To a great degree, the largest ethnic groups in China and the world is the Han Nationality (Chinese: 漢族). It constitutes the majority ethnic group among Chinese with approximately $91.59 \%$ of the total Chinese population of nearly 1159.4 million. According to the 2011 Chinese census, the Hui population is the second largest ethnic minority group with approximately 10.6 million (1.6\%) of the total Chinese population (Dillon, 1999; Pew Research Center, 2009; Wang, 2015). Demographically, whereas Mandarin is the main spoken language of the Hui people, as it is for Han as well, the most distinct differences between the two nationalities are their belief in different religions, traditional dietary habits, and social traditions. These three major differences are mutually exclusive, as Hui Muslims practice their religious requirement of 
consuming only "halal food" such as butchering of animals for food consumption in a religiously prescribed strict manner, as well as adhering to a myriad of other Islamic-mandated dietary codes and social commandments (Newman, 2004). According to Islamic jurisprudence, Muslims should refrain from drinking any quantity of alcoholic beverages and are prohibited from consuming any pork; pork products; the blood of any animal; and other animal types of meat such as meat from beasts or dead animals (Counihan \& Van Esterik, 2012; Junru, 2011; Newman, 2004).

A considerable body of epidemiological research provided evidence of a strong connection between poor dietary habits and a myriad of noncommunicable chronic diseases (NCDs), more notably, in low- and middle-income developing countries leading to exceptionally high rates of disability and mortality (World Health Organization, 2011). During the last three decades, China has experienced fundamental nutrition transition, traditional dietary and physical activity level changes. Particularly, the accelerated pace of sprawling urbanization in Chinse cities has become a major sociodemographic phenomenon of growing ruralto-city migration that has contributed to making drastic lifestyle changes, as well as the emergence of dietrelated chronic diseases in the general Chinses population. Research by Popkin et al. (1993) demonstrated that China faced numerous challenges of under-nutrition that had existed for decades. In a separate, but related research, based on the 1991 China Health and Nutrition Survey (CHNS), Popkin (2014) pointed out that due to several drastic dietary changes, adoption of a nontraditional lifestyle, coupled with recent modernization across the socioeconomic strata in China, the prevalence of obesity and other chronic NCDs have increased as a major public health burden that has principally affected the low-income Chinese. Du and Keys (2015) argued that dietary habits play a critical role in the development of many NCDs such as certain types of cancer, cardiovascular disease, obesity, Type2 diabetes, and stroke. Du and Keys (2015) further explained that there is not much known about the effects of dietary habits on these growing lifestyle-related conditions in China, where the dietary patterns differ greatly from those of Western populations. Guan (2015) observed that there is a high prevalence of diseases among the ethnic minority groups in rural China (Wang, 2015) due to several factors among them social customs, culture, food consumption, and genetic backgrounds. Guan (2015) and Ahmed (2016) further added that the socioeconomic status of the rural ethnic groups is a major contributing factor to the health disparity between the Chinese minority ethnic groups and the majority Chinese Hans. Research by Fu, Wang, and Wang (2005) reported differences in food consumption patterns and nutrient intake were also found in groups divided in line with their gender, residence, and household income. More recent research by Mendez and Popkin (2005); Popkin (2006) provided further evidence of the connection between dietary changes and the increased incidents of degenerative diseases associated with the modern dietary changes introduced to the Chinese traditional eating habits. This was primarily caused by the prevalence of Western-style fast food eateries, sugary soft drinks, and low-cost cooking oils; coupled with significant decreases in refined carbohydrate food prices, increased access to supermarkets, and sprawling urbanization.

\section{Methods and Data Sources}

Survey Development and Procedures

This cross-sectional investigation was conducted in China during the summer of 2015. Respondents were 662 Chinese adults aged 18 years and older, including men $(n=356)$ and women $(n=306)$. Participants in the study responded to an adapted version of a paper-and-pencil self-administered food frequency, dietary history, and food consumption cross-sectional population survey. The questions were designed to collect data on habitual food and beverages consumption patterns, healthy food shopping practices, healthy cooking methods, healthy food preparation techniques (baking food vs. frying it), the number of full meals consumed each day, the time of the last meal consumed each day, and related health practices. The study employed a modified version of the National Cancer Institute Food Frequency Questionnaire (FFQ) and the Diet History Questionnaire (DHQ) (National Cancer Institute (NCI), 2007; Thompson et al., 2000). In addition to the above-stated dietary habits and related health practices, the 23-question questionnaire encompassed questions to collect relevant data from the respondents concerning their demographic characteristics, including level of education; marital status; employment status; family size based on the number of adults over 18 years of age and children under 18 years of age living in the same household; ethnic group affiliation; and gender identification. Respondents were randomly selected volunteers from four urban cities (Zhengzhou, Kaifeng, Xi'an, \& Jinan) located in the Yellow River Basin Central Plains, Northwestern, and Midwestern Provinces of China (Figure 1). 


\section{Data Analysis}

The Statistical Analysis Software (SAS) Package, Version 9.3 was employed to analyze the collected data. T-tests were performed for nominal (classification) variables vs. ordinal variables where the nominal variable had exactly two levels (e.g., gender). Chi-square test (mostly 2 by 2 ) analyses were utilized if both variables were nominal. Certain nominal data items with multiple levels were re-coded into one of two levels prior to performing a 2 by 2 Chi-square test $(\chi 2)$. Most of the analyses involved calculating $\mathrm{p}$ values and classifying any value of a test statistic with a p-value of less than an alpha level of 0.05 as statistically significant. In this study, the tests were two-tailed.

\section{Results}

\section{Demographic Characteristics}

Participants in our study were 662 volunteers randomly selected from four urban cities in three provinces cities in central and eastern China (Henan, Shaanxi, and Shandong) (Figure 1). Most of the respondents hailed from the Chinese Han Ethnic Nationality (71.54\%), whereas (28.46\%) hailed from the Chinses Hui Ethnic Nationality (Table 1).

Table 1

Summary of Key Results of the Demographic Characteristics, Food Consumption

Patterns, and Dietary-Related Health Practices by the Two Nations (Han vs. Hui)

\begin{tabular}{|c|c|c|}
\hline Variable & $\operatorname{Han}(\%)$ & Hui (\%) \\
\hline Total respondents $(\mathrm{N}=662)$ & 71.54 & 28.46 \\
\hline Male respondents & 54.07 & 60.11 \\
\hline Female respondents & 45.93 & 39.89 \\
\hline Marital status: Married & 37.01 & 22.96 \\
\hline Marital status: Single & 62.99 & 6.09 \\
\hline $\begin{array}{l}\text { Overall Level of education } \\
\text { (college vs. no college) }\end{array}$ & 62.53 & 15.93 \\
\hline $\begin{array}{l}\text { Fresh fruit shopping patterns } \\
\text { ( } 3 \text { or more times per week) }\end{array}$ & 49.45 & 55.03 \\
\hline $\begin{array}{l}\text { Habitual healthy food ingredient shopping (all the } \\
\text { time) }\end{array}$ & 54.27 & 58.52 \\
\hline $\begin{array}{l}\text { Habitual healthy food cooking } \\
\text { (all the time) }\end{array}$ & 52.08 & 58.19 \\
\hline $\begin{array}{l}\text { Habitual healthy food preparation } \\
\text { technique (baking vs. frying) }\end{array}$ & $\begin{array}{l}\text { b } 77.03 \\
\text { f } 22.97\end{array}$ & $\begin{array}{l}\text { b } 82.2 \\
\text { f } 17.80\end{array}$ \\
\hline $\begin{array}{l}\text { Habitual daily breakfast intake } \\
\text { Habitual typical drink intake } \\
\text { (water vs. all other beverages) }\end{array}$ & $\begin{array}{l}65.36 \\
67.94\end{array}$ & $\begin{array}{l}80.87 \\
60.56\end{array}$ \\
\hline $\begin{array}{l}\text { Number of meals each day } \\
\text { (three or more full meals per day) }\end{array}$ & 62.09 & 72.53 \\
\hline $\begin{array}{l}\text { Time of the last meal consumed } \\
\text { between }(17: 00-24: 00 \text { PM })\end{array}$ & 97.54 & 95.32 \\
\hline
\end{tabular}


The participants in our study responded to a paper-and-pencil 23-question modified selfadministered Food Frequency Questionnaire (FFQ). The respondents were randomly sampled from the cities of Zhengzhou ( $n=241,36.40 \%)$; Kaifeng $(n=264,39.88 \%)$; Xi'an $(n=85,12.84 \%)$; and Jinan $(n=72$, $10.88 \%$ ). As it can be observed in Table 1, the gender proportion of the respondents corresponding to their ethnic nationality (Han vs. Hui) indicated that the Han male respondents were (54.07\%), whereas the female respondents were $(45.93 \%)$. With respect to the Hui, the male respondents were $(60.11 \%)$, whereas the female respondents comprised of $(39.89 \%)$, there was no statistically significant difference in the gender representation of the Han vs. Hui respondents, $\chi^{2}(1, \mathrm{n}=620), p<.170$. The marital status results revealed that the Han married respondents constituted $(37.1 \%)$, whereas the single respondents comprised of $(62.99 \%)$. Regarding the Hui marital status, the married respondents were $(22.96 .1 \%)$, whereas the single respondents encompassed $(6.09 \%)$, there was no statistically significant difference in gender representation between the Han and Hui respondents, $\chi^{2}(1, \mathrm{n}=620), p<.170$.

As it can be seen in Table 1, respondents' overall level of education results indicated that the Han were more likely to have higher levels of education with $(62.53 \%)$, whereas the Hui were more likely to have lower levels of education with $(15.93 \%)$, there was statistically significant difference in the level of education between the Han and Hui Nationalities' respondents, $\chi^{2}(1, \mathrm{n}=641), p<.001$.

Table 2 depicts an itemization of all respondents' educational status based on the level of education they have received at the time of data collection. The results showed that $(22.05 \%)$ of all respondents completed a Bachelor's degree, (15.77\%) completed a Master's degree, (1.68\%) completed a Doctorate degree; (13.02\%) completed a high school or equivalent degree, $(12.25 \%)$ completed an associate degree, $(10.11 \%)$ received some or no college education, (4.90\%) received some high school education with no diploma, (1.99\%) received a trade; technical; or vocational training, (15.31\%) received K-8th grade education, and (2.91\%) did not receive any formal education. With respect to marital status and education level, the results disclosed that single respondents were more likely to be more educated with $(67.46 \%)$, whereas their married counterparts were likely to be less educated with $(27.27 \%)$, there was statistically significant difference in the education level of single and married respondents, $\chi^{2}(1, \mathrm{n}=581), \mathrm{p}<.001$

Table 2 .

Frequency distribution of the Level Education reported by all respondents

\begin{tabular}{lllll}
\hline Level of Education & $\begin{array}{l}\text { Frequency } \\
(n)\end{array}$ & Percent $(\%)$ & $\begin{array}{l}\text { Cumulative } \\
\text { Frequency }\end{array}$ & $\begin{array}{l}\text { Cumulative } \\
\text { Percent }(\%)\end{array}$ \\
\hline No Formal Education & 19 & 2.91 & 19 & 2.91 \\
K-8 Education & 100 & 15.31 & 119 & 18.22 \\
Some HS, No Diploma & 32 & 4.90 & 151 & 23.12 \\
HS Grad or Equivalent & 85 & 13.02 & 236 & 36.14 \\
Trade/Tech./Voc. & 13 & 1.99 & 249 & 38.13 \\
Training & 80 & 12.25 & 329 & 50.38 \\
Associate Degree & 66 & 10.11 & 395 & 60.49 \\
Some College, & $N o$ & 22.05 & 539 & 82.54 \\
$\begin{array}{l}\text { Degree } \\
\text { Bachelor's Degree }\end{array}$ & 144 & 15.77 & 642 & 98.32 \\
Master's Degree & 103 & 1.68 & 653 & 100.00 \\
Doctorate Degree & 11 & & &
\end{tabular}

Note. HS is high school, Tech. is technical, and Voc. is vocational.

With reference to the employment status of all respondents (Table 3), the results indicated that $(30.66 \%)$ of the employed respondents were the main wage earner in the household, $(29.62 \%)$ was the second wage earner in the household; $(6.10 \%)$ of them was full-time homemakers, and $(33.62 \%)$ of them did not earn any income. 
Table 3.

Frequency distribution of employment and household income status reported by all respondents

\begin{tabular}{lcccc}
\hline Family Role & Frequency & $\begin{array}{l}\text { Percent } \\
(\%)\end{array}$ & $\begin{array}{l}\text { Cumulative } \\
\text { Frequency }\end{array}$ & $\begin{array}{l}\text { Cumulative } \\
\text { Percent (\%) }\end{array}$ \\
\hline No Income & 193 & 33.62 & 193 & 33.62 \\
Full-Time Homemaker & 35 & 6.10 & 228 & 39.72 \\
Main Wage Earner & 176 & 30.66 & 404 & 70.38 \\
Second Wage Earner & 170 & 29.62 & 574 & 100.00 \\
\hline
\end{tabular}

Regarding the family structure responses presented in (Table 4), the results indicated that nearly half of the respondents (49.55\%) reported having no children under the age of 18 years old who are living in the same household, $(36.71 \%)$ reported having one child living at home, $(10.85 \%)$ having two children, $(1.45 \%)$ having three children, $(1.27 \%)$ having four children, and $(0.18)$ having six children under the age of 18 years old who were living in the same household.

Table 4.

The average family size and number of children living at home per each household

Children Under 18 Years Old Living at Home

\begin{tabular}{lllll}
\hline $\begin{array}{l}\text { Number of } \\
\text { Children-at-Home }\end{array}$ & $\begin{array}{l}\text { Frequency } \\
\text { Count }\end{array}$ & Percent (\%) & $\begin{array}{l}\text { Cumulative } \\
\text { Frequency }\end{array}$ & $\begin{array}{l}\text { Cumulative } \\
\text { Percent (\%) }\end{array}$ \\
\hline No Children & 274 & 49.55 & 274 & 49.55 \\
One Child & 203 & 36.71 & 477 & 86.26 \\
Two Children & 60 & 10.85 & 537 & 97.11 \\
Three Children & 8 & 1.45 & 545 & 98.55 \\
Four Children & 7 & 1.27 & 552 & 99.82 \\
Six Children & 1 & 0.18 & 553 & 100.00
\end{tabular}

\section{Dietary Behaviors, Food Consumption Patterns, and Related Health Practice Factors}

Our study examined the association between the demographic characteristics of the Han and Hui ethnic respondents and eight dietary habits, food consumption patterns, and related health practices, including frequency of fresh fruit shopping (three or more each per week); frequency of healthy food ingredients shopping (all the time); applying healthy food cooking methods (all the time); employing healthy food preparation techniques (baking food vs. frying it); habitual daily breakfast consumption; habitual beverage consumption (water vs. all other beverages); number of full meals consumed each day (three to four); and 
the time of the last meal consumed each day. One of the noteworthy results was the interaction between marital status, consumption of a daily breakfast, level of education, and fresh fruit shopping patterns. As it can be observed in Figure 2, married respondents who reported having a lower level of education had a significantly higher rate of habitually consuming daily breakfast with $(77.27 \%)$. In comparison, the single respondents reported a rate of $(61.62 \%)$.

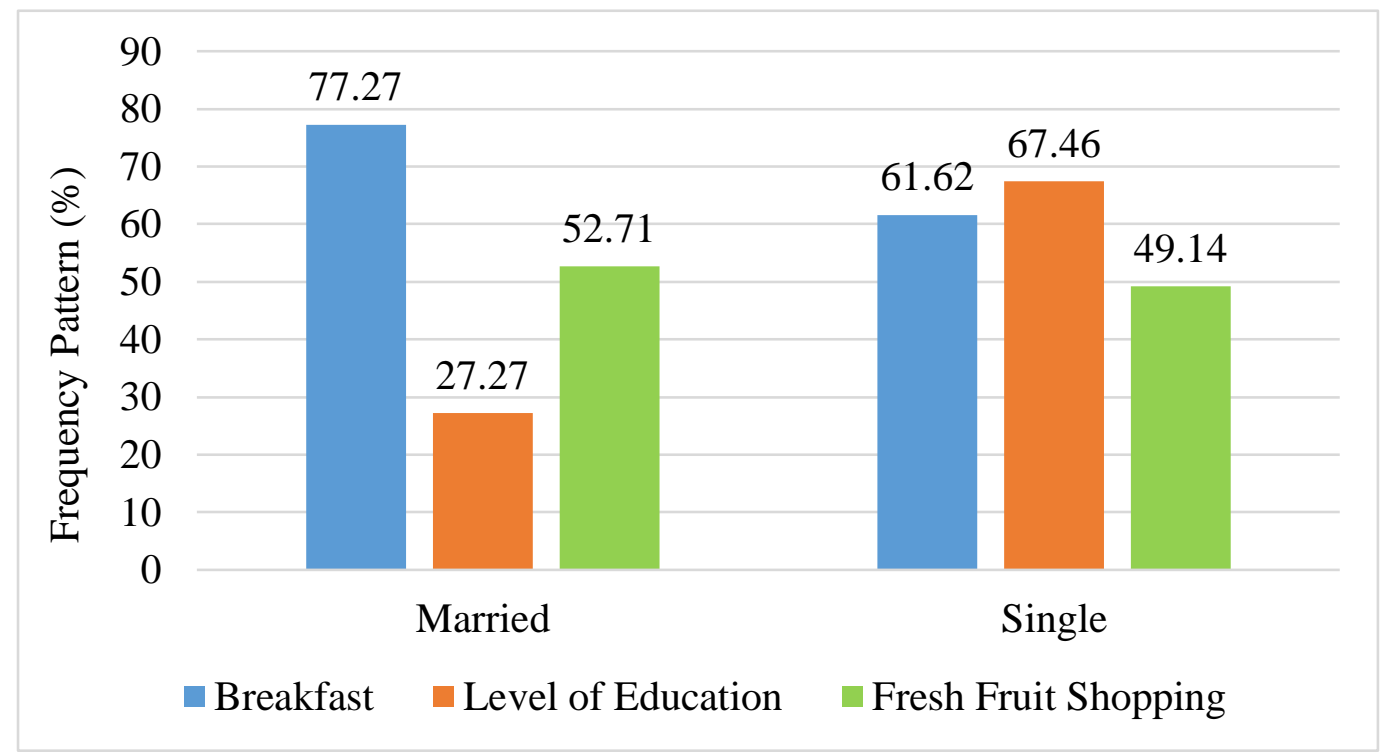

Figure 2. Daily breakfast consumption, education level, and fresh fruit shopping of three or more times per week patterns according to marital status.

As Figure 2 also demonstrates, the single respondents who reported a considerably higher level of college education $(67.46 \%)$ compared to their married counterparts' level of education of (27.27\%) were less likely to consume daily breakfast on a regular basis, there was a significant difference found between married and single respondents in their daily breakfast consumption habit, $\chi^{2}(1, \mathrm{n}=583), \mathrm{p}<.001$. Regarding the marital status and the frequency of fresh fruit shopping patterns (three or more each per week), the results indicated that married respondents reported a slightly higher rate of (52.71\%) above their single counterparts whose reported rate was $(49.14 \%)$, there was no significant difference between the married and single respondents in the frequency of fresh fruit shopping (three or more each per week), $\chi^{2}(1, \mathrm{n}=568), \mathrm{p}<0.395$.

As it can be viewed in Figure 3, the regular daily breakfast consumption habit results of the Han vs. Hui revealed that the Han respondents reported (65.36\%), compared to $(80.87 \%)$ rate recorded by the Hui, there was statistically significant difference in between the Han and Hui Nationalities, $\chi^{2}(1, \mathrm{n}=642), p<$ .001. The Hui were more likely to consume daily breakfast regularly than the Han. Similar findings were found in the frequency of fresh fruit shopping patterns according to nation affiliation (Han vs. Hui) presented in Figure 3, where the Hui respondents reported a slightly higher frequency of fresh fruit shopping pattern with $(55.03 \%)$ above their counterpart Hans with $(49.45 \%)$, there was no statistically significant difference between the two nations in fruit shopping, $\chi^{2}(1, \mathrm{n}=622), p<.215$. Figure 2 depicts the results from the regular daily breakfast consumption habit in connection with marital status, where the married respondents reported an intake rate of $(77.27 \%)$, compared to the single counterparts who reported $(61.62 \%)$ intake, there was a significant difference between married and single respondents in their daily intake of breakfast habit, $\chi^{2}(1, n=583), p<.001$. The dietary-related health practice results of the Han and Hui respondents are presented in Figure 4. The results showed that healthy food ingredient shopping patterns (all the time) by the Hui registered $(58.52 \%)$, followed closely by the Han with $(54.27 \%)$, no statistically significant difference was found between the two ethnic groups in their healthy food ingredient shopping pattern, $\chi^{2}(3, n=633), p$ $<.334$. Figure 4 further presents the results from the Han vs. Hui analysis for applying healthy food cooking methods (all the time), where the Hui came up with $(58.19 \%)$ and the Han reported (52.08\%), there was no statistically significant difference between the Han and Hui in their applying healthy food cooking method practice (all the time), $\chi^{2}(1, \mathrm{n}=634), p<.166$. 


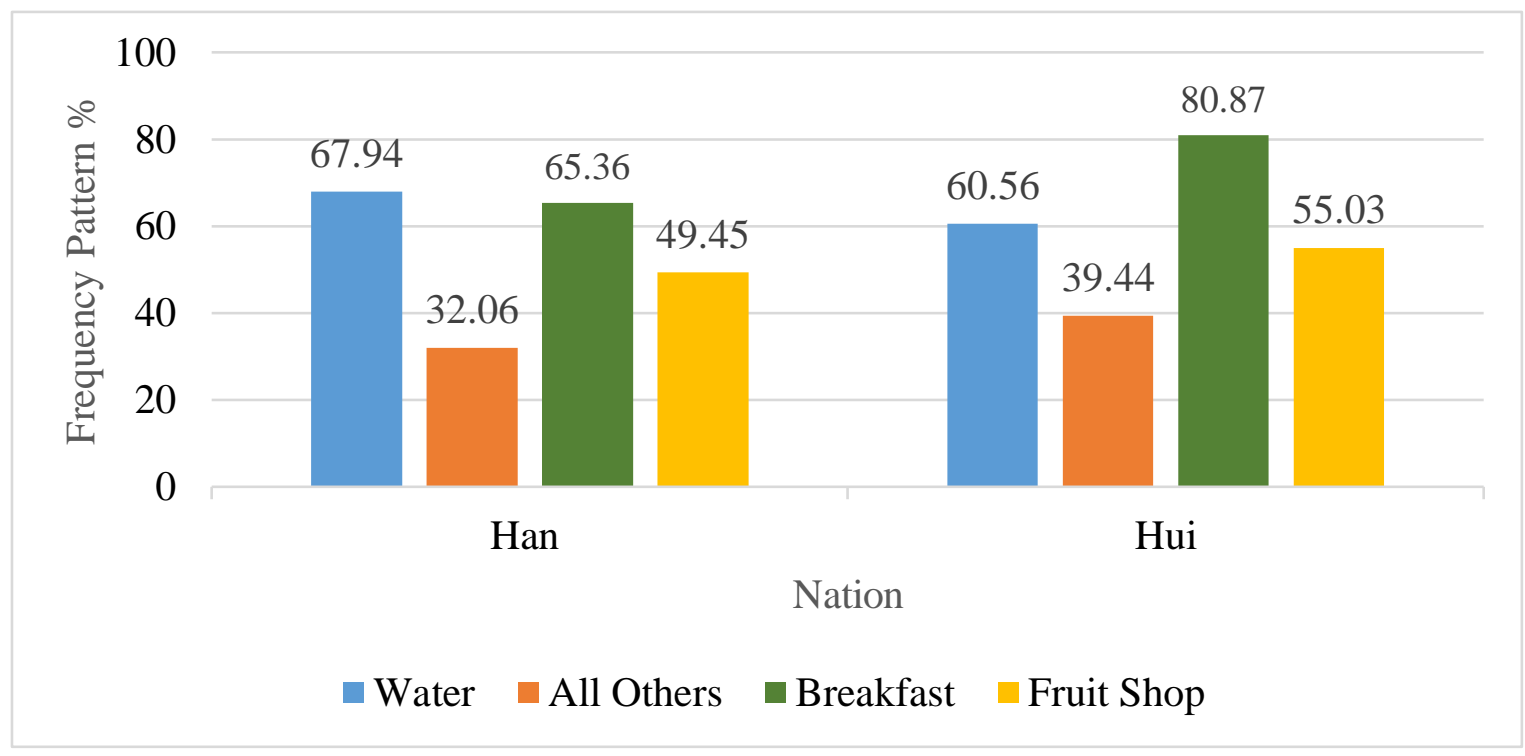

Figure 3. Habitual water intake vs. all other beverages, daily breakfast consumption, and fresh fruit shopping patterns (three or more times per week) by Nation (Han vs. Hui).

With reference to employing healthy food preparation techniques (baking food vs. frying it), the results portrayed in Figure 4 suggested that the Han were more likely to bake food with (77.03\%) and fry it with $(22.97 \%)$, whereas the Hui were more likely to bake food with $(82.2 \%)$ and fry it with $(17.80 \%)$. No statistically significant difference was found between Han and Hui in their practice of employing healthy food preparation techniques (baking vs. frying), $\chi^{2}(1, n=475), p<.237$. Figure 3 exhibits the results of water intake vs. all other beverages by the Han vs. Hui respondents, where Hans reported drinking water frequently by $(67.94 \%)$ as opposed to all other beverages with $(32.06 \%)$ frequency. The Hui recorded drinking water frequently with $(60.56 \%)$, compared to all other beverages with $(39.44 \%)$, there was no statistically significant difference found in the frequency of water intake vs. all other beverages between the Han and Hui respondents, $\chi^{2}(1, \mathrm{n}=482), p<.119$. With respect to the number of full meals consumed each day (three to four) by the Han vs. Hui respondent, the results indicated that the Hui respondents scored $(72.53 \%)$, whereas the Han respondents recorded $(62.09 \%)$, there was statistically significant difference between the Han and Hui in the consumption of three to four full meals each day, $\chi^{2}(1, \mathrm{n}=641), p<.012$. The Hui were more likely to consume three to four full meals each day than the Han. The findings of the time of the last meal consumed each day (17:00-24:00 PM) suggested that the Han and Hui respondents arrived at similar results, as the Han reported (97.54\%) and the Huis followed very closely with (95.32\%), there was no statistically significant difference found between the two ethnic groups regarding the time of the last meal consumed each day, $\chi^{2}(1, \mathrm{n}=577), p<.162$. The Han and Hui were more likely to consume the last meal of the day between the hours of 17:00-24:00 PM. 


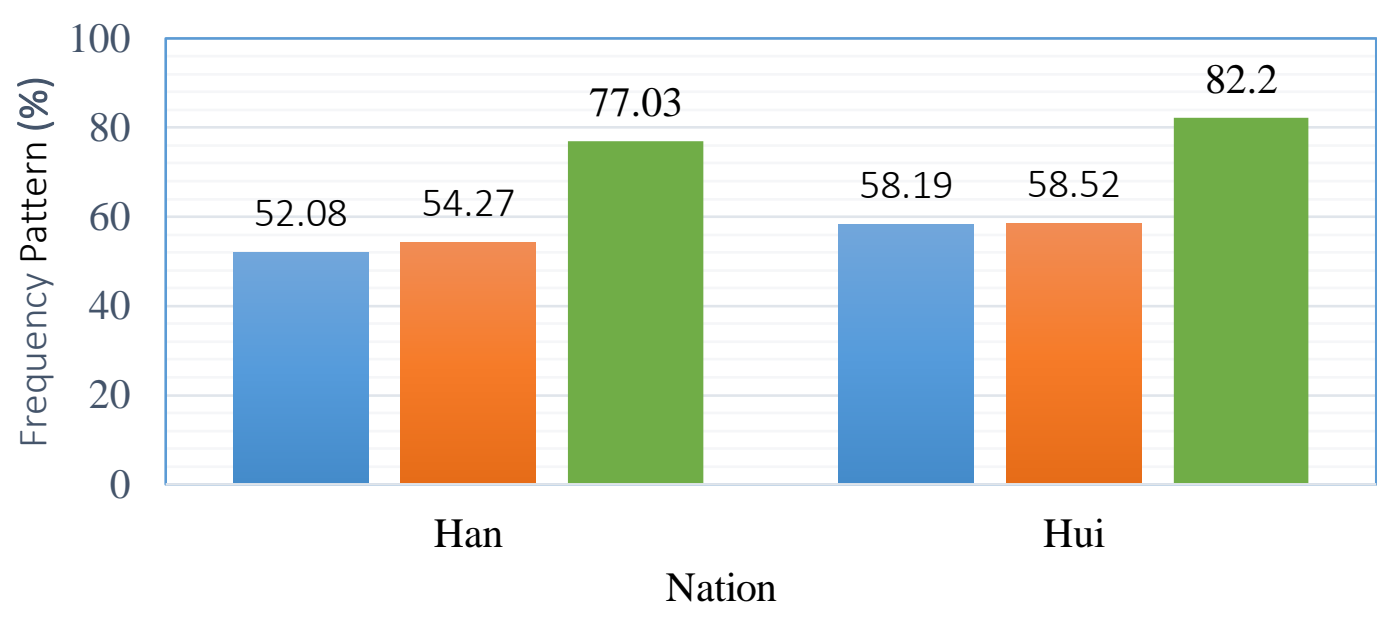

- H.F Cook. $\quad$ H.F Shop. $\quad$ H.F Prep.

Figure 4. Healthy food cooking (H.F Cook.), healthy food shopping (H.F Shop.), and healthy food preparation techniques (H.F Prep.) by baking vs. frying by Nation (Han vs. Hui).

\section{Discussion}

Our study was designed to examine the association between the demographic characteristics, food consumption patterns, and dietary-related health practices of the Chinese Han and Hui Ethnic Nationalities. Respondents of a sample population $(\mathrm{N}=662)$ answered a 23-question self-administered modified Food Frequency Questionnaire. The respondents were adults over the age of 18 years randomly selected from four urban Chinese cities (Zhengzhou, Kaifeng, Xi'an, \& Ji'nan). The results of our study revealed some key findings in some of the dietary habits, food and beverages intake patterns, and diet-related health practices. Similarly, notable findings were found in connection with the demographic characteristics of the two Chinese ethnic populations. The results of our study underscore that ethnicity, culture, and some demographic characteristics may possibly have a direct association with some of the dietary habits and food consumption patterns in ethnic groups.

\section{Demographic Characteristic}

With respect to the demographic Characteristics, the present study clearly provided several noteworthy findings. The Han Nationality participants accounted for the preponderance of the survey respondents $(71.54 \%)$, whereas the Hui Nationality made (28.46\%) of the respondents (Table 1). The sizable proportional difference between the Han and Hui respondents accurately reflects the actual proportionate difference in the general Chinese population, where the ethnic Han Nationality constitutes the preponderance of the mainland Chinese making approximately (91.59\%) of the total Chinese population. The Hui Nationality, on the other hand, comprises of approximately $1.6 \%$ of the total Chinese mainland population (Dillon, 1999; Pew Research Center, 2009).

The level of education results shown in (Table 1) indicated there was a significant difference in the education level between the Han and Hui respondents. Han respondents were more likely to be more educated in all levels of education with (62.53\%), whereas Hui respondents were more likely to be less educated in all levels of education with (15.93\%). In comparison, these findings are consistent with findings from a dietary patterns study comprised of (97\%) of Han Chinese respondents who reported that $(95.5 \%)$ of them completed at least primary education (Gao et al., 2013). With respect to the association between marital status and the level of education, the results revealed that the single respondents were more likely to be more educated with $(67.46 \%)$ than their married counterparts who reported only $(27.27 \%)$. This considerable difference can be reasonably explained by the fact that our study was conducted in large urban cities with major universities with sizable educated single student populations.

Marital status was one of the aims of this study. Summarized in Table 1, the findings revealed notable differences among the two ethnic groups. For the Hans, they had a considerably higher proportion of single respondents $(62.99 \%)$ compared with their married counterpart's rate of $(37.01 \%)$. This proportional 
difference can be reasonably explained by the fact that our study was conducted in major urban cities that have universities with large single student populations. In comparison, the Huis had a higher proportion of married respondents with $(22.96 \%)$, whereas their single counterparts consisted of $(6.09 \%)$. This considerable difference in the Hui marital status can be reasonably attributed to the fact that, traditionally, in the Hui Muslim culture, the Hui people are more likely to get married much younger than these of their Han counterparts.

Regarding the marital status factor in connection with family structure results (Tables 2 and 4), these results are consistent with the demographic characteristics of our study respondents. As we have indicated in the Results section, single respondents comprised of $(50.77 \%)$, whereas their married counterparts made up $(49.23 \%)$ of the total study respondents. This clearly explains the association between having a clear majority of the participants $(49.55 \%)$ who reported having no children; and having a clear majority of the respondents $(50.77 \%)$ who reported being single. By linking these results, it can be reasonably assumed that the single respondents have no children (Table 4). Furthermore, the second largest majority of participants $(36.71 \%)$ reported having one child only. This result is consistent with the one-child per family policy. This Chinese policy was introduced in 1979, as a mandate for the family planning program. In 2015, nevertheless, China decided to phase out this policy by early 2016 (Letcher, 2016). These results demonstrate that the study population represents a true a reflection of the family structure of the general Chinese population. Regarding the respondents who reported having 3-6 children living in the same households (Table 4), this can be reasonably explained by these respondents might have been living with an extended family, including children, parents, grandparents, and other relatives. In China, it is customary that all the extended family members would normally live in the same household. Traditionally, in China, family structure functions as one unit, where married sons; their wives; and their children live with the son's own parents in the same household providing care and support for the young children, as well as for the aging parents until those aging parents are deceased (Xu \& Xia, 2014).

The employment status was another aim of this study. Table 3 revealed that $(30.66 \%)$ of the employed respondents were the main wage earner in the household; $(29.62 \%)$ were the second wage earner in the household; $(6.10 \%)$ of them were full-time homemaker; and (33.62\%) of them did not earn any income. Compared with the other employment status categories, a possible explanation of the high rate of no income of (33.62\%), it may suggest that the respondents who did not earn an income were either full-time students, full-time homemakers, unemployed adults, or a mélange of all three employment status categories. One of the limitations of this study is that it did not examine if the employment status factors may have influenced the dietary habits, food consumption patterns, and related health behaviors of the two ethnic groups. However, these factors have been examined in a follow-up study conducted by us in China in 2016-2017.

\section{Dietary Habits, Food Consumption Patterns, and Related Health Practices}

One of the noteworthy findings we have obtained from the data analysis is the dietary patterns that both ethnic groups closely shared by reporting higher scores in consuming the last meal of the day between the hours of 17:00-24:00 PM. This late-night meal consumed by both ethnic groups is a clear indication of the fundamental changes in the eating patterns among the Chinse population during the last three decades. In part, this can be reasonably interpreted as one of the effects of adopting the Western culture eating behaviors and similar dietary habits. Especially in urban cities where our study was conducted, Chinese are increasingly disinclined to invest a good deal of time in food preparation and cooking. Consequently, the frequency of eating several meals in restaurants has increased markedly (Ma, 2015). Furthermore, due to the long history of socially amalgamating with each other, the Chinese ethnic groups, including the Hui Nationality have adopted some of the Han Nationality's eating behaviors such as consuming late-night meals. Whereas they still maintain many of their own ethnic traditions, the Hui people are celebrated as being the closest to and most assimilated ethnic group into the predominant Chinese Han's lifestyle and culture in China (Parker, 2016).

Another notable finding is the association between regularly consuming daily breakfast, being married, and the level of education. As it can be observed in Figure 2, married respondents who reported having a lower level of education had a significantly higher rate of consuming daily breakfast (77.27\%) than the single respondents $(61.62 \%)$. Contrary to our expectation, the single respondents who reported having a considerably higher level of education $(67.46 \%)$ than their married counterparts $(27.27 \%)$, were less likely to consume daily breakfast on a regular basis. A reasonable explanation for this finding could be it appears that having a higher level of education has not necessarily translated instinctively into adopting a healthy dietary behavior such as consuming daily breakfast on a regular basis by the single respondents. Moreover, 
we can infer that married couples were more likely to be influenced by the traditional Chinese culture of the importance of consuming daily breakfast for health reasons. They also might have been having more access to and interest in nutritional knowledge; especially, if they were having children as well. Our explanation is supported by research by Ma (2015) who maintained that residents in Beijing with (74.8\%), Shanghai with (86.8\%), and Guangzhou with $(90.5 \%)$ reported having breakfast every day. Ma (2015) further explained that the proportion of the residents who reported having daily breakfast on a regular basis aged $>35$ years old was higher than their counterparts aged $<35$ years old. Further, in this research, women were found to be more likely to consume breakfast with a higher consistent regularity than men. Residents who reported they did not consume breakfast regularly cited reasons for skipping eating breakfast, including lack of enough time, loss of appetite, and the desire to lose or maintain body weight. Irregular breakfast consumption was found to be a common dietary habit among nearly (25\%) of adolescent groups (Berkley, 2003; Yang et al., 2006). A further interpretation of the relationship between marital status, the level of education, and regular daily breakfast intake is that married respondents were more likely to place a greater health and social value of consuming breakfast every day as a family meal than their counterpart single respondents. Culturally, such food or a meal habit is described by Koctürk-Runefors (1990) as "value" food or a "value" meal. In addition, it could be possible that being single can be somewhat socially isolating from the habit of eating with other family members or groups. Sociologically, it appears that commensality plays a key role in promoting a social group, such as married couples, to eat their meals together more often than eating alone, including breakfast. Research by Eisenberg, et al. (2004) found that regular family meals may promote higher self-esteem, lower risk of depression, and have a better academic performance among adolescents. Several studies reported that daily breakfast consumption is associated with improving cognitive function related to memory, test grades, and school attendance. Breakfast as the first meal of the day provides the essential nutrients and energy needed for the day's physical and cognitive activities. The breakfast meal health benefits are associated with having improved overall quality of diet and daily essential nutrient intake. For these reasons, it is considered as the most important meal of the day (Al-Bashtawy, 2015; Nicklaus et al; Rampersaud et al., 2005). Rampersaud et al., 2005, stressed the importance of consuming a daily balanced breakfast regardless of age. Findings from psycho-behavioral studies suggested that children who consumed breakfast on a regular basis were more likely to get along well with their peers, as well as they would generally exhibit positive behavior during the school day than those children who do not regularly consume breakfast (Benton, Maconie, \& Williams, 2007; Phillips, 2005).

Figure 2 displays the findings obtained from the marital status differences in the fresh fruit shopping pattern scores (three or more times per week). The results revealed that there was a narrow statistical margin in the score differences between the married respondents who reported fresh fruit shopping pattern with $(52.71 \%)$, whereas single the respondents reported $(49.14 \%)$. Taking the narrow statistical margin into consideration, this result is in line with research findings related to consumer shopping behaviors and shopping patterns that showed most single consumers have a tendency not to purchase fresh fruits and vegetables as frequently or in large quantities as the average married couples, who are more likely to have children (Dehn, 2017; Steinhofer, 2005). Considering the statistically slim margin, our interpretation of this finding is that it is possible that both marital groups were most likely well informed of the health benefits of the consumption of fresh fruits. Furthermore, we also believe that because our study was conducted during the summer months where a wide variety of seasonal fruits are abundant at reasonable prices that might have contributed to increasing consumption rates by most of the singles and married respondents. This also might be the reason for the narrow the score differences between them. Our interpretation is consistent with a similar trend we have reported on in the Results section in connection with the Han and Hui ethnic groups. Research evidence supported the association between the health benefits of habitually consuming fresh fruits and nuts and preventing certain forms of cancer and cardiovascular disease (Hu et al., 1998; Jacobs, 1988).

With reference to ethnic differences in fresh fruit shopping patterns (three or more times per week) by the two ethnic groups, Hui respondents scored a frequency of $(55.03 \%)$ that is slightly higher than the Hans who reported (49.45\%). A reasonable explanation of the relative proximity of scores between than the Han and Hui respondents is we believe that was because of our study was conducted during the summer months where a wide variety of seasonal fruits are abundant at reasonable prices might have increased consumption patterns by most of the Han and Hui respondents. This can be also the reason for the narrow margin of the score difference between them. Our explanation is consistent with a similar trend we have described above in connection with the two marital status groups.

Table 4 presents the Hui vs. Han score differences for applying healthy food preparation techniques (i.e.., baking food vs. frying it). The results indicated that the Han were more likely to bake food with 
(77.03\%) and fry it with (22.97\%) rates, whereas the Hui were more likely to bake food with $(82.2 \%)$ and fry it with $(17.80 \%)$ rates. We consider baking food scores were higher than frying it is a positive dietaryrelated health behavior by both ethnic groups. The Hui respondents, however, reported proportionally higher scores for both baking food more and frying it less than the Han respondents. The possible explanation for the Hui reporting higher scores can be ascribed to the dietary practice that the Hui people follow specified Islamic dietary standards known "Halal food" that most of them observe, as we have described earlier in the Introduction section that "Halal food" is what the Hui people adhere to as Muslims when they purchase and prepare their food. Another reason that further explains baking food more than frying it by the Hui is that, culturally, Hui people have a preference for consuming wheaten-based foods. Noodles and noodle soups are the most popular staple foods in the Hui food culture. The second favorite is also wheat-based flour breadlike thick pancakes called in Chinese "dabing", and they are usually grilled or oven-baked (Gillette, 2000; Newman, 2004). Research by Koctürk-Runefors (1990) postulated that there a connection between food and cultural identity and sense of value. Koctürk-Runefors (1990) further explained that some foods that are deeply connected to a culture or an ethnic group carry more "value" to that culture or ethnic group; and therefore, it might be difficult to change or substitute overtime. In the Introduction section, studies by Ji et al. (1995) and Zhang, et al. (2002) provided epidemiological evidence on the increased risks of pancreatic and ovarian cancers among the residents of some Chinese cities were found to be linked to consuming deepfried foods and other animal fats. These findings were substantiated by several different but related epidemiological studies that suggested that a regular adequate consumption of fruits is linked to preventing certain forms of cancer and cardiovascular disease (Steinmetz \& Potter, 1996; Wang et al., 2014).

With respect to practicing healthy dietary behaviors practiced through the number of full meals consumed each day (three to four), the results showed that the Hui respondents reported (72.53\%), whereas their Han counterparts reported (62.09\%). This clearly indicates that a sizable proportion of each ethnic group considers consuming three to four full meals each day is an important dietary-related health practice. Similarly, we can draw an interpretation for water intake vs. all other beverages results reported by the Hans and Huis (Figure 3), where there was no significant difference between them, as both indicated preferring drinking water over any other beverages. This finding is consisted with what we have described above in the Introduction section, as water (Shui) is one of the five elements of well-being known as "wuxing" valued for good health in the Chinese culture. Whereas the Hui respondents reported a much lower level education of (15.93\%) (Table 1), they reported over 10\% higher rate than the Han in consuming three to four full meals each day). We can reasonably explain that these findings suggest that culturally, the Hui people are more likely to place further importance on consuming three to four full meals each day than their Han counterparts. This finding is consistent with what we have reported earlier regarding reporting similarly a higher rate of regularly consuming daily breakfast. As an ethnic group, consistent with Koctürk-Runefors's (1990) suggestion, it appears that the Hui people "value" consuming daily breakfast and three to four meals each day on a regular basis as a component of their cultural belief and ethnic identity, and not necessarily related to their level of education. To interpret these results holistically for both the Han and Hui combined, we can reasonably conclude that these data appropriately reflect the Chinese cultural tradition of placing a special importance on consuming at least three full square meals each day. Consistent with our interpretation of our results, Junru, 2011 suggested that for people from every Chinese nationality group, eating three regular meals per day (breakfast, lunch, and dinner) has been a standard practice of the Chinese traditional eating patterns since the Han Dynasty (206 BC-220 AD). This suggestion has been further supported by a more recent study by the China National Nutrition Survey (CNNS) that found Chinese people with different ethnic backgrounds might differ on the exact number of meals they consume each day; nevertheless, the majority $(94 \%)$ of Chinese consume three meals a day, whereas approximately $5 \%$ consume two meals each day. However, the number of meals may change from urban to rural areas. Approximately (25\%) of residents living in relatively poor rural areas consume two meals per day. On the other hand, in urban areas, where our study was conducted, residents usually consume 3 to 4 meals every day (Ma, 2015). This indicated that the data reported by the CNNS are consistent with the findings of our study.

\section{Conclusions}

The results of the present study identified noteworthy differences in the strength and association between the demographic characteristics, dietary habits, food consumption patterns, and related health practices between two of the major Chinese ethnic groups, the Han and Hi Nationalities. Although the present study generated strong evidence of some significant differences in the demographic characteristics such as the level education, marital status, ethnic group affiliation, and gender indicators; in part, the results similarly 
identified some strong associations between the dietary habits, food consumption patterns, related health practices, and the demographic characteristics such as regular daily breakfast intake and marital, water intake and ethnicity (Ahmet, 2016; Shah, 2015), frequent fresh fruit shopping patterns and marital status, and applying healthy food preparation techniques (frying food vs. baking it) and ethnicity. Consequently, we believe that the findings of this study offer evidence-based valuable information to be employed by public health policy makers, health educators, nutritionists, and healthcare professionals to develop initiatives and disseminate dietary-rated health behavior information to the Han, Hui ethnic groups, as well as the Chinese population at large. Even though our study examined the respondents' frequency of healthy food shopping and consumption patterns, this does not necessarily mean that these frequencies are translated into an actual intake of similar magnitudes. However, they may provide practical indicators of the actual consumption patterns. This study has also provided us with two useful insights. First, there a scarcity of published scholarly papers examining food consumption patterns and dietrelated health practices of the Chinese ethnic groups, especially the Hui Minority Nationality. Second, the degree of the interaction and strength of the association between the demographic factors, dietary habits, and food consumption patterns among the ethnocultural populations could be an intricate quantitative research investigation. These two insights would support the need for further future studies in, which would benefit this important field of research and the ethnic populations in China, as well as in other countries with culturally and ethnically diverse populations such in Europe and elsewhere.

\section{Declaration of Conflict of Interest}

The authors of this manuscript declare that they have no conflict of interest concerning its drafting, publication, or application.

\section{Acknowledgment}

We wish to express our gratitude to the Key Research Institute of Yellow River Civilization and Sustainable Development at Henan University, Kaifeng, Henan Province, China, the National Office of HighEnd Foreign Experts of the Chinese Ministry of Education, and the National Nature Science Foundation of China (Project Number: 41471104) for their sponsorship and support for this study. We also very much appreciate the assistance provided for the statistical analysis provided by Barry Walton of Millersville University, Millersville, PA, USA. We would also like to convey our special thanks to the Institute's graduate students Yuanyuan Cai, Fengmei Pan, Huixian Ji, and Yicun Lv for providing their invaluable assistance in collocating and systematizing the survey data. We affirm that the underwriters of our study had neither influenced nor played any role in the development, research design, data analysis, interpretation, or drafting of our manuscript.

\section{References}

Ahmet, M. (2016). Ethnicity, identity and group vitality: A study of Burushos of Srinagar. Journal of Ethnic and Cultural Studies, 3(1), 1-10.

Al-Bashtawy, M. (2015). Exploring the reasons why school students eat or skip breakfast. Nurs Child Young People, 27(6), 16-22. doi:10.7748/ncyp.27.6.16.e622.

Benton, D., Maconie, A., \& Williams, C. (2007). The influence of the glycemic load of breakfast on the behavior of children in school. Physiol Behav, 92(4), 717-24.

Berkley, C. S., Rockett, H. R., Gillman, M. W., Field, A. E., \& Colditz, G. A. (2003). A longitudinal study of skipping breakfast and weight change in adolescents. International Journal of Obesity, 27, 12581266.

Chang, K. C. (1977). Food in Chinese culture:Aanthropological and historical perspectives. New Haven, CT: Yale University Press.

Counihan, C., \& Van Esterik, P. (2012). Food and culture: A reader third edition. (3rd ed.). Oxford, UK: Routledge.

Damgaci, F. K. (2016). Vatandaşlık, Çeşitlilik ve Çoğulculuğun Tarihsel Süreç İçerisinde Türk Eğitim Sistemindeki Yeri. 21. Yüzyllda Eğitim ve Toplum, 5(13), 223-238.

Dehn, M. (2017). Cross-cultural study of food and CPG products in a new market: Case study in the United States. Thailand. (Honors Theses) (pp. 2821-2822). Western Michigan University, Kalamazoo, MI.

Dillon, M. (1999). China's Muslim Hui community: migration, settlement, and sects. London, UK: Curzon Press 
Du, H., \& Keys, T. (2015). Diet and health: The China Kadoorie Biobank (CKB) Study of Chronic Disease. Oxford, UK: University of Oxford Publication.

Eisenberg, M. E., Olson, R., Neumark-Sztainer, D., Story, M., \& Bearinger, L. H. (2004). Correlations between family meals and psychosocial well-being among adolescents. Archives of Pediatrics and Adolescent Medicine, 158, pp. 792-796.

Faltis, C. (2014). Toward a race radical vision of bilingual education for Kurdish users in Turkey: Commentary. Journal of Ethnic and Cultural Studies, 1(1), 1-5.

Feagin, J. R., \& Feagin, C. B. (2007). Racial and ethnic relations (8th ed.). Upper Saddle River, NJ: Prentice Hall.

Fu, J., Wang, Z., \& Wang, B. (2005). Transitions of food groups and nutrients in the northeast of China: A 3-year-interval's follow-up study. The Internet Journal of Nutrition and Wellness, 2 (02), pp. 1-8.

Gao, H., Stiller, C. K., Scherbaum, V., Biesalski, H. K., Wang, Q., Hormann, E., \&. Bellows, A. C. (2013). Dietary intake and food habits of pregnant women residing in urban and rural areas of Deyang City, Sichuan Province, China. Nutrients, 5, 2933-2954. doi:10.3390/nu5082933.

Gillette, M. B. (2000). Between Mecca and Beijing: Modernization and consumption among urban Chinese Muslims. Redwood City, CA: Stanford University Press.

Gladney, D. C. (1998). Ethnic identity in China: The making of a Muslim minority nationality. Fort Worth, TX: Harcourt Brace College.

Guan, M. (2015). Ethnic difference of disease prevalence in rural China: Examples and Explanations. Health, 7, 449-455. Retrieved from http://dx.doi.org/10.4236/health.2015.74052.

Henderson, J. (2002). Wu Xing (Wu-hsing): Five phases. In Cua, A.S. Editor (Ed). Encyclopedia of Chinese Philosophy (pp. 686-688). New York: Routledge.

Hu, F. B., Stampfer, M. J., Manson, J. E., Rimm, E. B., Colditz, G. A., Rosner, B. A., \& Speizer, F. E. (1998). Frequent nut consumption and risk of coronary heart disease in women: prospective cohort study. $B M J, 2(3), 317: 1341-5$. doi:dx.doi.org/10.1136/bmj.317.7169.1341.

Jacobs, L. R. (1988). Fiber and colon cancer. Gastroenterology Clinics of North America, 17, pp. 747-760.

Ji, B.T., Chow, W. H., Gridley, G., McLaughlin, J. K., Dai, Q., Wacholder, S., Hatch, M. C., Gao, Y. T., \& Fraumeni, J. F. (1995). Dietary factors and the risk of pancreatic cancer: A case-control study in Shanghai, China. Cancer Epidemiol Biomarkers Prev, 4(8), 885-93.

Junru, L. (2011). Chinese food. Cambridge, UK: Cambridge University Press.

Kittler, P. G., Sucher, K., \& Nelms, N. M. (2012). Food and culture (6th ed.). Boston, MA: Wadsworth.

Kocktürk-Runefors, T. (1990). Changes in food habits and nutritional status of immigrants from Turkey in Sweden. Bibl Nutr Dieta, 45, 157-164.

Letcher, K. (2016). One-child policy: Chinese Government program. Encyclopedia Britannica, https://www.britannica.com/topic/one-child-policy

Lin, K. (2000). Chinese food cultural profile. EthnoMed. Seattle, WA: University of Washington Press, https://ethnomed.org/clinical/nutrition/chinese_food_cultural_profile

Lipman, J. N. (1998). Familiar Strangers: A History of Muslims in Northwest China. Seattle, WA: University of Washington Press.

Ma, G. (2015). Food, eating behavior, and culture in Chinese society. Journal of Ethnic Foods, 2(4), 195199. doi:http://dx.doi.org/10.1016/j.jef.2015.11.004.

Mendez, M. A., \& Popkin B. M. (2005). Globalization, urbanization and nutritional change in the developing world. Electronic of J Agric Dev Econ, 1, 220-241. Retrieved from ftp://ftp.fao.org/docrep/fao/007/ae225e/ae225e00.pdf

National Cancer Institute: Division of Cancer Control and Population Sciences (2007). Diet history questionnaire paper-based forms, http://epi.grants.cancer.gov/DHQ/forms/

Newman, J. M. (2004). Islamic cuisine in China: Chinese ethnic minorities and their foods. Institute for the Advancement of the Science and Art of Chinese Cuisine, 11(2), 7-35.

Nicklaus, T. A., Reger, C., Myers, L., \& O'Neil, C. (2002). Breakfast consumption with and without vitaminmineral supplement use favorably impacts daily nutrient intake of ninth-grade students. J Adolesc Health, 27, 314-321.

Olson, J. S. (1998). An Ethnohistorical Dictionary of China. Westport, CT: Greenwood.

Parker, J. (2016, October 6). The Hui: China's other Muslims. The Economist, http://www.economist.com/news/china/21708274-choosing-assimilation-chinas-hui-have-becomeone-worlds-most-successful-muslim 
Pew Research Center (2009). Mapping the global Muslim population, http://www.pewforum.org/2009/10/07/mapping-the-global-muslim-population/

Phillips, G. W. (2005). Does eating breakfast affect the performance of college students on biology exams? Bioscene: Journal of College Biology Teaching, 30(4), 15-19.

Popkin, B. M. (2006). Global nutrition dynamics: the world is shifting rapidly toward a diet linked with noncommunicable diseases. Am J Clin Nutr, 84(2), 289-298.

Popkin, B. M. (2014). Synthesis and implications: China's nutrition transition in the context of changes across other low and middle-income countries. Obesity Reviews: An Official Journal of the International Association for the Study of Obesity, 15(1), 1110-1111.

Popkin, B. M., Keyou, G., Zhai, F., Guo, X., Ma, H., \& Zohoori, N. (1993). The nutrition transition in China: a cross-sectional analysis. Eur J Clin Nutr, 47(5), 333-46.

Rampersaud, G. C., Pereira, M. A., Girard, B. L., Adams, J., \& Metzl, J. D. (2005). Breakfast habits, nutritional status, body weight, and academic performance in children and adolescents. Journal of the American Dietetic Association, 105(5), 743-760. doi:org/10.1016/j.jada.2005.02.007.

Roberts, J. A. G. (1999). A concise history of China. New Haven, CT: Harvard University Press.

Shah, M. (2016). Ethnicity based social exclusion of Nomads in Khyber District Malakand, PakhtunkhwaPakistan. Journal of Ethnic and Cultural Studies, 3(1), 11-19.

Steinhofer, K. (2005). Young singles: A look at the grocery shopping preferences of a unique and underestimated market, survey \& analysis. (Honors Theses) (pp. 4-5). Western Michigan University, Kalamazoo, MI.

Steinmetz, K. A. \& Potter, J. D. (1996). Vegetables, fruit, and cancer prevention: A review. Journal of the American Dietetic Association, 96(10), 1027-1039. doi:org/10.1016/S0002-8223(96)00273-8.

Tarman, B., \& Gürel, D. (2017). Awareness of social studies teacher candidates on refugees in Turkey. The Journal of Social Studies Research. Retrieved from http://dx.doi.org/10.1016/j.jssr.2016.11.001i

Thompson, F. E., Kipnis, V., Krebs-Smith, S. M., Kahle, L. L., Midthune, D., Potischman, N., \& Schatzkin, A. (2000). Evaluation of 2 brief instruments and a food-frequency questionnaire to estimate daily number of servings of fruit and vegetables. Am J Clin Nutr, 71(6), 1503-1510.

Valera, S. (2008). Food in Chinese culture. Asia Society Publication. Retrieved from http://asiasociety.org/blog/asia/food-chinese-culture?page $=0,5$

Wang, G. (2015). Han Chinese proportion in China's population drops: 2011 census data (2012-4-28). Xinhua News (English). Retrieved from http://news.xinhuanet.com/english2010/china/201104/28/c_13849933.htm

Wang, T. (2015). Marginality of rural migrant students in eleven Chinese high schools. Journal of Ethnic and Cultural Studies, 2(2), 21-32.

Wang, X., Ouyang, Y., Liu, J., Zhu, M., Zhao, G., Bao, W., \& Hu, F.B. (2014). Fruit and vegetable consumption and mortality from all causes, cardiovascular disease, and cancer: Systematic review and dose-response meta-analysis of prospective cohort studies. BMJ, 349. doi:dx.doi.org /10.1136/bmj.g4490.

World Health Organization (2011). Global status report on noncommunicable diseases. Geneva: WHO, http://www.who.int/nmh/publications/ncd report2010/en/

$\mathrm{Xu}, \mathrm{A} \& \mathrm{Xia}, \mathrm{Y}$. (2014). The Changes in mainland Chinese families during the social transition: A critical analysis. Faculty Publications. Department of Child, Youth, and Family Studies, Lincoln, NE, University of Nebraska Press, (pp. 31-49). http://digitalcommons.unl.edu/famconfacpub/91/

Yang, R.J., Wang, E.K., Hsieh, Y.S., \& Chen, M. Y. (2006). Irregular breakfast eating and health status among adolescents in Taiwan. BMC Public Health, 6(1), 295. doi:10.1186/1471-2458-6-295.

Zhang, M., Yang, Z. Y., Binns, C. W., \& Lee, A. H. (2002). Diet and ovarian cancer risk: A case-control study in China, Br J Cancer, 86(5), 712-717. 